\title{
A review: Oxidative stress and its role in reproduction
}

\author{
Galina Nenkova, Albena Alexandrova* \\ Laboratory of Free Radical Processes, Bulgarian Academy of Sciences, Sofia, Bulgaria \\ Email: ${ }^{*}$ a_alexandrova_bas@yahoo.com
}

Received 18 October 2012; revised 27 November 2012; accepted 14 January 2013

\begin{abstract}
Recently the problem of infertility has become more acute. About $3 \%-7 \%$ of couples suffer a lasting infertility problem and there is a tendency of constant increasing of this number. Existing data reveal that in many of the infertility cases, the cause of sterility is the disabled sperm as a result of the oxidative stress (OS). There are several sources of reactive oxygen species (ROS) in the sperm. Both enzymatic and nonenzymatic antioxidants act as a counteraction against ROS production in seminal plasma. However due to the limited cytoplasm, the spermatozoa are more susceptible to oxidative damage because of the lack of protective cytoplasmic antioxidant enzymes. Currently, many couples suffering fertility problems resort to assisted reproductive technology (ART). Unfortunately, the success rate of ART is relatively low-about $30 \%$. Sperm processing incl. Centrifugation, freezing and thawing is associated with the generation of ROS. A range of strategies for sperm prevention of $O S$ are developed. Many studies concern antioxidants and their impact on sperm function, but there are a lot of contradictions in terms of doses and types of combinations that can be used in ART. Future studies should aim the creation of standard and reliable operating procedures for increasing the successfulness of these techniques. This paper offers an overview of the impact of $O S$ on the reproduction, pointing out the sources of ROS in the sperm, the antioxidant defense systems, and the strategies for prevention and improvement of the sperm reproductive functions, incl. those applied in ART.
\end{abstract}

Keywords: Antioxidants; Assisted Reproductive Technology; Reactive Oxygen Species; Sperm

\section{THE PROBLEM OF INFERTILITY}

Recently the problem of infertility has become more acute, affecting couples from all over the world. According to official statistics, the number of childless

*Corresponding author. couples in Bulgaria is about 270,000, i.e. almost every sixth family in this country is affected. One of each seven couples in the UK suffers reproductive problems. In Sweden, about $10 \%$ of the couples desiring children are infertile (according to data of the Sahlgrenska University Hospital). About $10 \%$ of the women aged 18 44 in the USA (6.1 million) experience difficulties in getting pregnant (according to The Centers for Disease Control and Prevention of Disease (CDC, 2011). Pooled data reveal that worldwide between three and seven per cent of all couples suffer a lasting infertility problem (Report of The Department of Reproductive Health and Research at WHO, 2010). The trend shows that this number will increase in the future as a result of the effect of several factors, namely unhealthy lifestyle with stress, use of substandard food, smoking, early and indiscriminate sex and related diseases, environmental pollution, etc. The planned postponement of pregnancy for later in life when fertility usually declines is also an influential factor. For women aged 35 years, about 94\% of those wishing to conceive become pregnant within 3 years, while in women aged 38 years this percentage decreases to $77 \%$ within 5 years.

Currently, many couples suffering fertility problems resort to assisted reproductive technology (ART), one of which is the in vitro method-the last and only hope for sterile couples. The fact that the 2010 Nobel Prize for Medicine and Physiology was awarded to the creator of the in vitro procedure the British professor Robert Edwards demonstrates that his discovery affects a large part of humankind. Unfortunately, the success rate of ART is relatively low about $30 \%$.

\section{REPRODUCTION AND OXIDATIVE STRESS}

All aspects of life, incl. reproduction are affected by reactive oxygen species (ROS). ROS are free radicals (FR), which play a crucial role in the physiological process of spermatogenesis, as well as in capacitation, hyperactivation and penetration of the sperm [1-3], oocyte maturation and regression of corpus luteum during normal ovarian reproductive cycle in women. 
However, upon overproduction of ROS or in case of reduced ability of the organism to protect against them, they can trigger pathological processes in male and female reproductive system [4-6]. The status, when ROS levels exceed the capacity of body antioxidant system and cause damage to cells, tissues or organs is referred to as oxidative stress (OS) [7-9]. According to existing data, the cause of sterility in $30 \%-80 \%$ of infertility cases is sperm that is disabled as a result of OS [10]. OS causes sterility by several mechanisms: 1) ROS damage sperm membrane (containing a large number of polyunsaturated fatty acids, which are vulnerable to ROS attacks-the socalled lipid peroxidation), leading to decreased motility and difficult fusion between sperm and oocyte [11-13]; 2) ROS mitochondrial damage, which reduces the energy available in the cell and thus impedes the movement of sperm $[3,14,15]$. Impaired motility causes a smaller number of sperm reaching the egg, which in turn greatly reduces the likelihood of fertilization [16,17]; 3) ROS damage sperm DNA. They are capable of directly attacking both purine and pyrimidine bases and the sugarphosphate backbone. Under normal conditions, sperm DNA is tightly packed with the participation of protamines, and this protects it from the attack by FR. During spermatogenesis histones are initially displaced by transition proteins and then by protamines. Protamines are significantly smaller than histones and contain many positively charged amino acid residues. This allows DNA strands which are highly negatively charged to wrap tightly around the protamine molecules. Moreover protamines contain cysteine residues, allowing formation of disulfide bonds between adjacent protamine molecules. This is a prerequisite for the formation of a very stable nucleoprotamine complex in which DNA is protected from the adverse effects of extrinsic and intrinsic factors. Although most of the human sperm chromatin is packaged by protamine, about $15 \%$ of the DNA remains packaged by histones. Histones bind to specific DNA sequences. The chromatin packaged by histones, is less tightly compacted and therefore more likely to be damaged by FR [18,19]. In infertile men a protamine deficiency is observed, which makes the genetic material of their germ cells more sensitive to elevated ROS levels [19]. DNA sequences that are packaged by histones are less tightly compacted and can easily decondense during fertilization. Presumably these sequences are involved in early embryo development [19]. It is found that one of the main reasons for the poor development of the blastocyst is the damaged paternal DNA [20]. Deletions, mutations and other genetic defects induced by FR, [21,22] automatically affects the DNA of the newly formed embryo. In normal pregnancy or normal IVF (not ICSI) the oxidative changes affecting sperm membrane will block fertilization, which in turn will not allow damaged genetic material to be involved in the formation of the embryo. In procedures such as IVF-ICSI, however (because of the microinjection) it is possible that sperm with significantly impaired DNA could fertilize the corresponding eggs [23]. Although most of the resulting embryos will die at blastocyst stage or early fetal development, some of them can survive. As a result, children with damaged DNA originating from the father, will be born. Consequences of the birth of such children are not yet sufficiently clarified, but suggest greater likelihood of developing genetic diseases and cancer in childhood [24,25].

\section{SOURCES OF ROS IN SEMEN}

There are two main sources of FR in the spermleukocytes and immature spermatozoa [26]. Of these, leukocytes are considered as the main source of ROS [27]. The majority of semen samples contain leukocytes, and the vast majority of leukocytes are neutrophils and macrophages. Their function is associated with excessive generation of ROS, which ultimately leads to dysfunction of sperm [28-34]. Almost every human ejaculate contains leukocytes, which makes it difficult to detect ROS produced by the sperm. In order to prove that spermatozoa (not just leukocytes) are responsible for ROS production, male germ cells are separated from seminal leukocytes using density gradient centrifugation. It is found that significant amounts of ROS are generated in the spermatozoa fraction, too. The ability of sperm to produce ROS is inversely proportional to their maturity. During spermatogenesis male germ cells lose much of their cytoplasm to acquire their distinctive slender and elongated shape. In immature teratozoosperm forms often a surplus of cytoplasmic residues is observed. These residues are rich in the enzyme glucose-6phosphate dehydrogenase, which controls the flow of glucose to the cell and hence the synthesis of betaNADPH-reduced form (NADP(H)) (via hexose monophosphate pathway). NADP(H)-oxidase, located in the membrane of the sperm, uses $\mathrm{NADP}(\mathrm{H})$ and generates ROS [29]. Therefore the teratozoosperm male germ cells produce higher ROS levels compared to normal spermatozoa. This exerts a negative effect on sperm quality [35]. The prolonged incubation of semen samples containing high levels of immature sperm before their processing increases the risk of OS damage of the mature spermatozoa [36].

Mitochondria are another important source of ROS [37]. According to literature data [38] about $1 \%-3 \%$ of oxygen reduced in mitochondria, generate superoxide anion radicals $\left(\mathrm{O}_{2}^{-}\right)$. There are at least nine key points in the mitochondria, where this is possible [39]: Complex I (aka NADH-ubiquinone oxidoreductase, C-I), complex 
III (aka bc1 complex, ubiquinone: cytochrome c reductase, C-III), cytochrome b5 reductase, monoamine oxidase (MAO-A and MAO-B, EC 1.4.3.4), dihydroorotate dehydrogenase (DHOH, EC 1.3.3.1 or EC 1.3.99.11), $\alpha$-glycerophosphate dehydrogenase (aka glycerol-3-phosphate dehydrogenase, aka mGPDH, EC 1.1.99.5), succinate dehydrogenase (SDH, aka succinate: ubiquinone oxidoreductase, complex II, EC 1.3.5.1), akonitaza (mitochondrial (m-) aconitase, EC 4.2.1.3), $\alpha$-ketoglutarate dehydrogenase complex (KGDHC, aka 2-oxoglutarate dehydrogenase).

Xanthine oxidase, a key enzyme in purine catabolism, is also involved in the production of ROS in sperm [40,41]. Cellular adenosine triphosphate (ATP) is dephosphorylated to adenosine monophosphate (AMP), which is degraded to hypoxanthine. ATP depletion leads to the loss of ATP-dependent transmembrane ion transport and results in disturbances in ion homeostasis. Passive transport of ions across cell membranes is performed: $\mathrm{K}^{+}$and $\mathrm{Mg}^{2+}$ diffuse outward the cell and $\mathrm{Na}^{+}, \mathrm{Ca}^{2+}$ - inward the cell. One of the most important consequences of increased $\mathrm{Ca}^{2+}$ cellular content is the activation of Cadependent protease that cleaves the xanthine dehydrogenase to xanthine oxidase. The accumulated hypoxanthine converts to xanthine and uric acid. The reaction is catalyzed by xanthine oxidase, which in the presence of oxygen generates $\mathrm{O}_{2}^{-}$.

\section{ANTIOXIDANT PROTECTION}

There are three main enzymatic antioxidant systems in seminal plasma: superoxide dismutase (SOD), catalase (CAT) and glutathione peroxidase/reductase system. SOD and CAT eliminate $\mathrm{O}_{2}^{-}$and $\mathrm{H}_{2} \mathrm{O}_{2}$, respectively, transforming them into $\mathrm{O}_{2}$ and $\mathrm{H}_{2} \mathrm{O}$. Glutathione peroxidases (GSH-Px1-5) reduce peroxides using glutathione (GSH) as a donor of electrons. The activity of GSH-Px is determined by the regeneration of reduced glutathione, which is carried by the enzyme glutathione reductase (GSSG-Red). It is found that selective inhibitors of this enzyme prevent the recovery of reduced glutathione, resulting in decreased activity of GSH-Px in male germ cells and thus enhance oxidative stress [42]. Balance and interaction between GSH-Px, GSSG-Red and glutathione play an important role in protecting sperm from oxidative damage.

In addition, there is a number of other non-enzymatic components in the seminal fluid with antioxidant effect such as: vit. C, vit. E [43], vit. D [44], urate [45], albumin, glutathione [46] taurine, hypotaurin [47].

While seminal plasma is rich in antioxidants [48], sperm contain a limited cytoplasm amount and therefore the amount of cytoplasmic enzymes in them is scanty. In ART the seminal plasma is removed, leading to in- creased susceptibility of sperm to oxidative damage due to the lack of protective systems. One of the main reasons for the removal of plasma is the presence of motility inhibitor derived from semenogelin I and II [49] in it, as well as because of the leukocytes [50] which, as already mentioned, are the major source of ROS. Centrifugation [51] and the process of freezing and thawing of semen [52] are associated with the generation of ROS. Bilodeau et al. [53] found that ROS generated during the cycles of freezing-thawing are decisive for the function of spermatozoa and that the levels of antioxidants decrease with each pass of such a cycle.

\section{STRATEGIES TO PROTECT SPERM FROM THE ROS}

A range of strategies for the prevention of oxidative stress, which leads to reduction and/or permanent impairment of reproductive functions, are developed. They include changes in living habits, e.g. reduction and cessation of smoking, change of dietary regime, directed to intake of foods rich in antioxidants, incl. vitamins and minerals, prevention or reduction the exposure to contaminated environment at work or at home, etc.

ART is an alternative for childless couples in case of sperm fertility disturbance. It is experimentally shown that the use of antioxidants has a positive effect on the processing and storage of semen. Different antioxidants are applied: chelators or free radicals scavengers. Chelators, deactivates the metal (mainly iron) ions which catalyze the generation of hydroxyl radicals $(\mathrm{OH})$ via the socalled Fenton reaction [31]. Currently it is assumed that $\mathrm{OH}$ are the main damaging agent. They initiate lipid peroxidation (LPO) by decomposition of peroxides in peroxyl and alkoxyl radicals, which in turn drive the chain reaction of LPO. Transferrin, lactoferrin and ceruloplasmin as endogenous metal chelators in human sperm control the LPO of the plasma membrane, preserving its integrity [54]. Metal chelators exert beneficial effects against oxidative DNA modifications. It has been shown in in vitro experiments with salmon sperm that use of metal chelators (ethylene diamine tetraacetic acid (EDTA), 1, 10 phenanthroline and neocuproine) reduces DNA damage [55]. Such studies, however, are not made with human sperm, except those with addition of EDTA [51]. In vitro addition of sulphydryl chelators such as 2.3 dimerkaptopropan-1-sulfonate and meso-2, 3-dimerkaptosuccinate enhance the quality of semen during ART [50]. Another sulphydryl-containing agent-dithiothreitol (DTT) according to some authors [56] is a good antioxidant and added to the storage medium keeps the quality of the spermatozoa. However, other authors [57] report that DTT initiates sperm decondensation with subsequent changes in size and morphology. Some che- 
lators such as nitrosonaftol, o-fenantroline, sodium diethyldithiocarbamate, cupferron, hydroxyquinoline are toxic to animal sperm [58].

In regard to the ROS scavengers and their role in maintaining and improving the quality of sperm there is a number of studies examining the sperm supplementation with antioxidant enzymes, vitamins and other substances. Literature data, however, are very controversial. Some authors have shown that the addition of catalase (CAT) prevents the reduction of sperm motility after subjecting them to OS [59,60], while others have found no beneficial effect of CAT on sperm motility [51,61]. Treatment with superoxide dismutase (SOD) has also given contradictory results. According to Rossi et al. [60], the in vitro addition of SOD prevents the sperm plasma membrane from LPO and helps to restore their quality after freezing-thawing procedures. However, Baumberg et al. [59] show that the addition of SOD increased DNA fragmentation in equine sperm, rather than reducing the OS effects. Similar discrepancies exist for the addition of vitamin E, glutathione, hypotaurine and many other antioxidants.

It appears that the concentration of the applied antioxidants is very important. For example, low CAT concentrations (1 and $10 \mathrm{U}$ ) increase the rate of acrosome reaction in contrast to the high concentration (100 U). EDTA in a concentration of $10 \mu \mathrm{M}$ reliably increases sperm motility, but applied in a concentration of $1 \mathrm{mM}$ leads to a sharp drop in motility to near zero [51].

The combination of different antioxidants may also have diverse effects. In vitro applied ascorbic acid (vitamin C) has a high antioxidant capacity associated with the interruption of free-radical chain reaction in seminal plasma [62]. Alpha tocopherol (vitamin E) also possesses antioxidant activity. It has been shown that it inhibits sperm LPO in in vitro experiments [63]. Supplied individually both vitamins prevent DNA damage, but together have an opposite effect [64]. The concomitant intake of vitamin C (350 mg/day) and vitamin E (250 $\mathrm{mg} /$ day) in vivo can prevent DNA damage in sperm after ejaculation [65]. In infertile patients with high levels of oxidative DNA damage, the combination of vitamin C and $\mathrm{E}$, together with glutathione results in a slight increase in the concentration of sperm. Genistein and equol (isoflavones) in combination have a strong protective effect against DNA damage than applied separately [66]. Perhaps there is a very narrow physiological range in which these antioxidants can work synergistically.

In conclusion, numerous studies concern antioxidants and their impact on sperm function, but there are many contradictions in terms of doses and types of combinations that can be used in ART. Further studies should aim to the creation of standard and reliable operating procedures to increase the success of these techniques and to avoid eggs fertilization by impaired sperm, as well as subsequent development of embryos with damaged DNA.

\section{REFERENCES}

[1] Aitken, R.J., Ryan, A.L., Baker, M.A. and McLaughlin, E.A. (2004) Redox activity associated with the maturation and capacitation of mammalian spermatozoa. Free Radical Biology and Medicine, 36, 994-1010. doi:10.1016/j.freeradbiomed.2004.01.017

[2] Allamaneni, S.R., Naughton, C.K., Sharma, R.K., Thomas Jr., A.J. and Agarwal, A. (2004) Increased seminal reactive oxygen species levels in patients with varicoceles correlate with varicocele grade but not with testis size. Fertility and Sterility, 82, 1684-1686. doi:10.1016/j.fertnstert.2004.04.071

[3] de Lamirande, E., Tsai, C., Harakat, A. and Gagnon, C. (1998) Involvement of reactive oxygen species in human sperm acrosome reaction induced by A23187, lysophosphatidylcholine and biological fluid ultrafiltrates. Journal of Andrology, 19, 585-594.

[4] Bankson, D.D., Kestin, M. and Rifai, N. (1993) Role of free radicals in cancer and atherosclerosis. Clinics in Laboratory Medicine, 13, 463-480.

[5] Hietanen, E., Bartsch, H., Bereziat, J.C., Camus, A.M., McClinton, S., Eremin, O., Davidson, L. and Boyle, P. (1994) Diet and oxidative stress in breast, colon and prostate cancer patients: A case-control study. European Journal of Clinical Nutrition, 48, 575-586.

[6] Agarwal, A. and Saleh, R.A. (2002) Role of oxidants in male infertility: Rationale, significance, and treatment. Urologic Clinics of North America, 29, 817-827. doi:10.1016/S0094-0143(02)00081-2

[7] Moller, P., Wallin, H. and Knudsen, L.E. (1996) Oxidative stress associated with exercise, psychological stress and life-style factors. Chemico-Biological Interactions, 102, 17-36. doi:10.1016/0009-2797(96)03729-5

[8] Sharma, R.K. and Agarwal, A. (1996) Role of reactive oxygen species in male infertility. Urology, 48, 835-850. doi:10.1016/S0090-4295(96)00313-5

[9] Saleh, R.A., Agarwal, A., Nada, E.A., El-Tonsy, M.H., Sharma, R.K., Meyer, A., Nelson, D.R. and Thomas, A.J. (2003) Negative effects of increased sperm DNA damage in relation to seminal oxidative stress in men with idiopathic and male factor infertility. Fertility and Sterility, 79, 1597-1605. doi:10.1016/S0015-0282(03)00337-6

[10] Agarwal, A., Sharma, R.K., Nallella, K.P., Thomas Jr., A.J., Alvarez, J.G. and Sikka, S.C. (2006) Reactive oxygen species as an independent marker of male factor infertility. Fertility and Sterility, 86, 878-885.

[11] Agarwal, A., Hamamah, S. and Shekarriz, M. (1994) Reactive oxygen species and fertilizing capacity of spermatozoa. Contracept Fertil Sex, 22, 327-330.

[12] Kobayashi, H., Gil-Guzman, E., Mahran, A.M., Rakesh, K., Nelson, D.R., Thomas Jr., A.J. and Agarwal, A. (2001) Quality control of reactive oxygen species measurement 
by luminol-dependent chemiluminescence assay. Journal of Andrology, 22, 568-574.

[13] Zalata, A.A., Ahmed, A.H., Allamaneni, S.S., Comhaire, F.H. and Agarwal, A. (2004) Relationship between acrosin activity of human spermatozoa and oxidative stress. Asian Journal of Andrology, 6, 313-318.

[14] de Lamirande, E. and Gagnon, C. (1992) Reactive oxygen species and human spermatozoa. II Depletion of adenosine triphosphate plays an important role in the inhibition of sperm motility. Journal of Andrology, 13, 379386.

[15] de Lamirande, E., Jiang, H., Zini, A., Kodama, H. and Gagnon, C. (1997) Reactive oxygen species and sperm physiology. Reviews of Reproduction, 2, 48-54. doi:10.1530/ror.0.0020048

[16] Whittington, K., Harrison, S.C., Williams, K.M., Day, J.L., McLaughlin, E.A., Hull, M.G. and Ford, W.C. (1999) Reactive oxygen species (ROS) production and the outcome of diagnostic tests of sperm function. International Journal of Andrology, 22, 236-242. doi:10.1046/j.1365-2605.1999.00174.x

[17] Kao, S.H., Chao, H.T., Chen, H.W., Hwang, T.I., Liao, T.L. and Wei, Y.H. (2007) Increase of oxidative stress in human sperm with lower motility. Fertility and Sterility, 89, 1183-1190. doi:10.1016/j.fertnstert.2007.05.029

[18] Oliva, R. (2006) Protamines and male infertility. Human Reproduction Update, 12, 417-435. doi:10.1093/humupd/dml009

[19] O’brien, J. and Zini, A. (2005) Sperm DNA integrity and male infertility. Urology, 65, 16-22. doi:10.1016/j.urology.2004.07.015

[20] Seli, E., Gardner, D.K., Schoolcraft, W.B., Moffatt, O. and Sakkas, D. (2004) Extent of nuclear DNA damage in ejaculated spermatozoa impacts on blastocyst development after in vitro fertilization. Fertility and Sterility, 82, 378-383. doi:10.1016/j.fertnstert.2003.12.039

[21] Moustafa, M.H., Sharma, R.K., Thornton, J., Mascha, E., Abdel-Hafez, M.A., Thomas Jr., A.J. and Agarwal, A. (2004) Relationship between ROS production, apoptosis and DNA denaturation in spermatozoa from patients examined for infertility. Human Reproduction, 19, 129-138. doi:10.1093/humrep/deh024

[22] Tominaga, H., Kodama, S., Matsuda, N., Suzuki, K. and Watanabe, M. (2004) Involvement of reactive oxygen species (ROS) in the induction of genetic instability by radiation. Journal Radiation Research (Tokyo), 45, 181188. doi:10.1269/jrr.45.181

[23] Zorn, B., Vidmar, G. and Meden-Vrtovec, H. (2003) Seminal reactive oxygen species as predictors of fertilization, embryo quality and pregnancy rates after conventional in vitro fertilization and intracytoplasmic sperm injection. International Journal of Andrology, 26, 279285. doi:10.1046/j.1365-2605.2003.00424.X

[24] Aitken, R.J. and Krausz, C. (2001) Oxidative stress, DNA damage and the Y chromosome. Reproduction, 122, 497506. doi:10.1530/rep.0.1220497

[25] Aitken, R.J., Baker, M.A. and Sawyer, D. (2003) Oxidative stress in the male germ line and its role in the aetiol- ogy of male infertility and genetic disease. Reproductive BioMedicine Online, 7, 65-70.

http://www.ncbi.nlm.nih.gov/pubmed/12930576 doi:10.1016/S1472-6483(10)61730-0

[26] Garrido, N., Meseguer, M., Simon, C., Pellicer, A. and Remohi, J. (2004) Pro-oxidative and anti-oxidative imbalance in human semen and its relation with male fertileity. Asian Journal of Andrology, 6, 59-65.

[27] Aitken, R.J., Buckingham, D., West, K., Wu, F.C., Zikopoulos, K. and Richardson, D.W. (1992) Differential contribution of leucocytes and spermatozoa to the generation of reactive oxygen species in the ejaculates of oligozoo-spermic patients and fertile donors. Journal of Reproduction and Fertility, 94, 451-462. doi:10.1530/jrf.0.0940451

[28] Aitken, R.J. and Baker H.W. (1995) Seminal leukocytes: Passengers, terrorists or good samaritans? Human Reproduction, 10, 1736-1739.

[29] Aitken, R.J., Fisher, H.M., Fulton, N., Gomez, E., Knox, W., Lewis, B. and Irvine, S. (1997) Reactive oxygen species generation by human spermatozoa is induced by exogenous NADPH and inhibited by the flavoprotein inhibitors diphenylene iodonium and quinacrine. Molecular Reproduction and Development, 47, 468-482. doi:10.1002/(SICI)1098-2795(199708)47:4<468::AID-M RD14>3.0.CO;2-S

[30] Hendin, B.N., Kolettis, P.N., Sharma, R.K., Thomas Jr., A.J. and Agarwal, A. (1999) Varicocele is associated with elevated spermatozoal reactive oxygen species production and diminished seminal plasma antioxidant capacity. Journal of Urology, 161, 1831-1834. doi:10.1016/S0022-5347(05)68818-0

[31] Ochsendorf, F.R. (1999) Infections in the male genital tract and reactive oxygen species. Human Reproduction Update, 5, 399-420. doi:10.1093/humupd/5.5.399

[32] Pasqualotto, F.F., Sharma, R.K., Potts, J.M., Nelson, D.R., Thomas, A.J. and Agarwal, A. (2000) Seminal oxidative stress in patients with chronic prostatitis. Urology, 55, 881-885. doi:10.1016/S0090-4295(99)00613-5

[33] Saleh, R.A. and Agarwal, A. (2002) Oxidative stress and male infertility: From research bench to clinical practice. Journal of Andrology, 23, 737-752.

[34] Sharma, R.K., Pasqualotto, A.E., Nelson, D.R., Thomas Jr., A.J. and Agarwal, A. (2001) Relationship between seminal white blood cell counts and oxidative stress in men treated at an infertility clinic. Journal of Andrology, 22, 575-583.

[35] Said, T.M., Agarwal, A., Sharma, R.K., Mascha, E., Sikka, S.C. and Thomas Jr., A.J. (2004) Human sperm superoxide anion generation and correlation with semen quality in patients with male infertility. Fertility and Sterility, 82, 871-877. doi:10.1016/j.fertnstert.2004.02.132

[36] Gil-Guzman, E., Ollero, M., Lopez, M.C., Sharma, R.K., Alvarez, J.G., Thomas Jr., A.J. and Agarval, A. (2001) Differential production of reactive oxygen species by subsets of human spermatozoa at different stages of maturation. Human Reproduction, 6, 1922-1930. doi:10.1093/humrep/16.9.1922 
[37] Koppers, A., De Iuliis, G., Finnie, J., McLaughlin, E. and Aitken, R.J. (2008) Significance of mitochondrial reactive oxygen species in the generation of oxidative stress in spermatozoa. Journal of Clinical Endocrinology and Metabolism, 93, 3199-3207. doi:10.1210/jc.2007-2616

[38] Halliwell, B. and Gutteridge, J.M.C. (2007) Free radicals in biology and medicine. IV Edition, Oxford Press, Oxford, 26.

[39] Andreyev, A., Kushnareva, Y. and Starkov, A. (2005) Mitochondrial metabolism of reactive oxygen species. Biochemistry (Moscow), 70, 200-214. Translated from Biokhimiya, 70, 246-264.

[40] Aitken, R.J., Buckingham, D. and Harkiss, D. (1993) Use of a xanthine oxidase free radical generating system to investigate the cytotoxic effects of reactive oxygen species on human spermatozoa. Journal of Reproduction and Fertility, 97, 441-450. doi:10.1530/jrf.0.0970441

[41] Sanocka, D., Miesel, R., Jedrzejczak, P. and Kurpisz, M.K. (1996) Oxidative stress and male infertility. Journal of Andrology, 17, 449-454.

[42] Alvarez, J.G. and Storey, B.T. (1989) Role of glutathione peroxidase in protecting mammalian spermatozoa from loss of motility caused by spontaneous lipid peroxidation. Gamete Research, 23, 77-90. doi:10.1002/mrd.1120230108

[43] Sies, H., Stahl, W. and Sundquist, A.R. (1992) Antioxidant functions of vitamins. Vitamins E and C, beta-carotene, and other carotenoids. Annals of the New York Academy of Sciences, 669, 7-20. doi:10.1111/j.1749-6632.1992.tb17085.x

[44] Jensen, M.B., Bjerrum, P.J., Jessen, T.E., Nielsen, J.E., Joensen, U.N., Olesen, I.A., Petersen, J.H., Juul, A., Dissing, S. and Jørgensen, N. (2011) Vitamin D is positively associated with sperm motility and increases intracellular calcium in human spermatozoa. Human Reproduction, 26, 1307-1317. doi:10.1093/humrep/der059

[45] Lewis, S.E., Sterling, E.S., Young, I.S. and Thompson, W. (1997) Comparison of individual antioxidants of sperm and seminal plasma in fertile and infertile men. Fertility and Sterility, 67, 142-147. doi:10.1016/S0015-0282(97)81871-7

[46] Garrido, N., Meseguer, M., Alvarez, J., Simón, C., Pellicer, A. and Remohí, J. (2004) Relationship among standard semen parameters, glutathione peroxidase/glutathione reductase activity, and mRNA expression and reduced glutathione content in ejaculated spermatozoa from fertile and infertile men. Fertility and Sterility, 82, 1059-1066. doi:10.1016/j.fertnstert.2004.04.033

[47] Holmes, R., Goodan, H., Shihabi, Z. and Jarow, J. (1992) The taurine and hypotaurine content of human semen. Journal of Andrology, 13, 289-292.

[48] Gallardo, J.M. (2007) Evaluation of antioxidant system in normal semen. Revista de Investigación Clínica, 59, 4247.

[49] Yoshida, K., Yamasaki, T., Yoshiike, M., Takano, S., Sato, I. and Iwamoto, T. (2003) Quantification of seminal plasma motility inhibitor/semenogelin in human seminal plasma. Journal of Andrology, 24, 878-884.
[50] Henkel, R.R. and Schill, W.B. (2003) Sperm preparation for ART. Reproductive Biology and Endocrinology, 14, 108. doi:10.1186/1477-7827-1-108

[51] Chi, H.J., Kim, J.H., Ryu, C.S., Lee, J.Y., Park, J.S., Chung, D.Y., Choi, S.Y., Kim, M.H., Chun, E.K. and Roh, S.I. (2008) Protective effect of antioxidant supplementation in sperm-preparation medium against oxidative stress in human spermatozoa. Human Reproduction, 23, 1023-1028. doi:10.1093/humrep/den060

[52] Di Santo, M., Tarozzi, N., Nadalini, M. and Borini, A. (2012) Human sperm cryopreservation: update on techniques, effect on DNA integrity, and implications for ART. Advances in Urology, Article ID: 854837, 12 Pages.

[53] Bilodeau, J.F., Chatterjee, S., Sirard, M.A. and Gagnon, C. (2000) Levels of antioxidant defenses are decreased in bovine spermatozoa after a cycle of freezing and thawing. Molecular Reproduction and Development, 55, 282-288. doi:10.1002/(SICI)1098-2795(200003)55:3<282::AID-M $\underline{\mathrm{RD} 6>3.0 . \mathrm{CO} ; 2-7}$

[54] Sanocka, D. and Kurpisz, M. (2004) Reactive oxygen species and sperm cells. Reproductive Biology and Endocrinology, 23, 12. doi:10.1186/1477-7827-2-12

[55] Bruskov, V.I., Malakhova, L.V., Masalimov, Z.K. and Chernikov, A.V. (2002) Heat-induced formation of reactive oxygen species and 8-oxoguanine, a biomarker of damage to DNA. Nucleic Acids Research, 30, 1354-1363. doi:10.1093/nar/30.6.1354

[56] Lindemann, C., O’Brien, J. and Giblin, F. (1988) An investigation of the effectiveness of certain antioxidants in preserving the motility of reactivated bull sperm models. Biology of reproduction, 38, 114-120. doi:10.1095/biolreprod38.1.114

[57] Huszar, G., Celik-Ozenci, C., Cayli, S., Kovacs, T., Vigue, L. and Kovanci, E. (2004) Semen characteristics after overnight shipping: Preservation of sperm concentrations, HspA2 ratios, CK activity, cytoplasmic retention, chromatin maturity, DNA integrity, and sperm shape. Journal of Andrology, 25, 593-604.

[58] White, D.R. (1985) Studies of the spermicidal activity of chelating agents. Australian Journal of Biological Sciences, 8, 387-395.

[59] Baumber, J., Ball, B.A. and Linfor, J.J. (2005) Assessment of the cryopreservation of equine spermatozoa in the presence of enzyme scavengers and antioxidants. American Journal of Veterinary Research, 66, 772-779. doi:10.2460/ajvr.2005.66.772

[60] Rossi, T., Mazzilli, F., Delfino, M. and Dondero, F. (2001) Improved human sperm recovery using superoxide dismutase and catalase supplementation in semen cryopreservation procedure. Cell Tissue Bank, 2, 9-13. doi:10.1023/A:1011592621487

[61] Calamera, J.C., Fernandez, P.J., Buffone, M.G., Acosta, A.A. and Doncel, G.F. (2001) Effects of long-term in vitro incubation of human spermatozoa: Functional parameters and catalase effect. Andrologia, 33, 79-86. doi:10.1046/j.1439-0272.2001.00409.X

[62] Lewis, S.E.M., Sterling, E.S.L., Young, I.S. and Thompson, W. (1997) Comparison of individual antioxidants of 
sperm and seminal plasma in fertile and infertile men. Fertility and Sterility, 67, 142-147.

doi:10.1016/S0015-0282(97)81871-7

[63] Aitken, R.J. and Clarkson, J.S. (1988) Significance of reactive oxygen species and antioxidants in defining the efficacy of sperm preparation techniques. Journal of Andrology, 9, 367-376.

[64] Sharma, R.K., Said, T. and Agarwal, A. (2004) Sperm DNA damage and its clinical relevance in assessing reproductive outcome. Asian Journal of Andrology, 6, 139148.

[65] Hughes, C.M., Lewis, S.E., McKelvey-Martin, V.J. and Thompson, W. (1998) The effects of antioxidant supple- mentation during Percoll preparation on human sperm DNA integrity. Human Reproduction, 13, 1240-1247. doi:10.1093/humrep/13.5.1240

[66] Sierens, J., Hartley, J.A., Campbell, M.J., Leathem, A.J. and Woodside, J.V. (2002) In vitro isoflavone supplementation reduces hydrogen peroxide-induced DNA damage in sperm. Teratogenesis, Carcinogenesis, and Mutagenesis, 22, 227-234.

http://www.ncbi.nlm.nih.gov/pubmed/11948633 $\underline{\text { doi:10.1002/tcm.10015 }}$ 